\title{
Achondroplasia in the Premature Infant: An Elusive Diagnosis in the Neonatal Intensive Care Unit
}

\author{
Kimberly E. Fagen, MD ${ }^{1,2}$ Anna R. Blask, MD² Eva I. Rubio, MD ${ }^{2}$ Dorothy I. Bulas, MD² \\ ${ }^{1}$ Department of Radiology, Walter Reed National Military Medical \\ Center, Bethesda, Maryland \\ ${ }^{2}$ Department of Radiology, Children's National Health System, \\ Washington, District of Columbia \\ Address for correspondence Kimberly E. Fagen, MD, Department of \\ Radiology, Walter Reed National Military Medical Center, 8901 \\ Wisconsin Avenue, Bethesda, MD 20889 \\ (e-mail: kimberly.e.fagen.mil@mail.mil; \\ ablask@childrensnational.org).
}

Am J Perinatol Rep 2017;7:e8-e12.

Achondroplasia is a difficult prenatal diagnosis to make before the late second and third trimester. We describe two cases where an infant was born prematurely with no overt signs of achondroplasia. Despite multiple chest and abdominal radiographs during the neonatal course, the diagnosis was not made until term equivalent age was reached. We retrospectively reviewed these two cases to highlight the elusive findings of achondroplasia in the premature infant.
Achondroplasia is the most common nonlethal, short-limbed skeletal dysplasia comprising 5 to $15 / 100,000$ births. $^{1-3}$ The cause of this skeletal dysplasia is most commonly a de novo mutation in the transmembrane domain of the fibroblast growth factor receptor-3, which affects the cartilaginous growth plate, disturbing function during growth. ${ }^{2}$ The inheritance pattern is autosomal dominant if a parent bears the FGFR3 mutation. The homozygous form is lethal and has similar features to thanatophoric dysplasia. ${ }^{2}$ Prenatal diagnosis of heterozygous achondroplasia can be difficult until the third trimester when limb lengths, particularly of the humeri and femora, begin to demonstrate a significant drop in interval growth as manifest on fetal growth charts. Drop off in the limb lengths can less commonly be observed in the late second trimester. Additional sonographic findings in utero include frontal bossing, increased head circumference relative to long bone measurements, short fingers, and polyhydramnios but these are often not remarkable at the 20-week screening ultrasound (US). ${ }^{1}$ We describe the postnatal course and delayed recognition of radiographic findings in two infants born prematurely without overt signs of achondroplasia until near-term equivalent age, mirroring the difficulty of early prenatal in-utero diagnosis. We were unable to find similar cases reported in the literature.

\section{Case Reports}

Case 1: This infant was born prematurely at $23^{+4}$ weeks' gestation at an outside hospital due to maternal hypertension, preeclampsia, and HELLP syndrome (hemolysis, elevated liver enzymes, low platelet count). At 31 days of life, the infant was transferred to our neonatal intensive care unit (NICU) due to multiple medical problems related to prematurity including a patent ductus arteriosus, bronchopulmonary dysplasia, and necrotizing enterocolitis (NEC). During the course of her NICU stay, she also developed germinal matrix, intraventricular and cerebellar hemorrhages with mild hydrocephalus as evaluated by head sonograms and magnetic resonance imaging (MRI). A gastrostomy tube was placed for nutritional support. NEC necessitated bowel resection and additional complications of prematurity included chronic lung disease and asthma.

No skeletal abnormalities were initially recognized on multiple portable newborn chest and abdomen radiographs or on physical examination throughout the NICU course. The patient was discharged from the NICU to a chronic care facility due to the intensity of ongoing care requirements.

After discharge to home, at 7 months of life (corrected age of 3 months) both the infant's mother and pediatrician became increasingly concerned about her short stature and requested a genetics evaluation. She was small in all of her growth parameters, but especially in her height. Assessment by a geneticist revealed relative macrocephaly, short nose with depressed bridge, anteverted nares, short extremities, and widening of the third and fourth finger interdigital space (trident hand). Skeletal survey (- Fig. 1A-G) to evaluate for the possibility of a skeletal dysplasia was performed and demonstrated frontal bossing, rhizomelic shortening with flared metaphyses, received

April 5, 2016

accepted after revision

August 2, 2016
DOI http://dx.doi.org/

$10.1055 / \mathrm{s}-0036-1592188$.

ISSN 2157-6998.
Copyright $\odot 2017$ by Thieme Medical Publishers, Inc., 333 Seventh Avenue, New York, NY 10001, USA. Tel: +1(212) 584-4662.
License terms

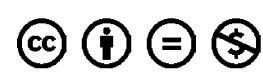


A
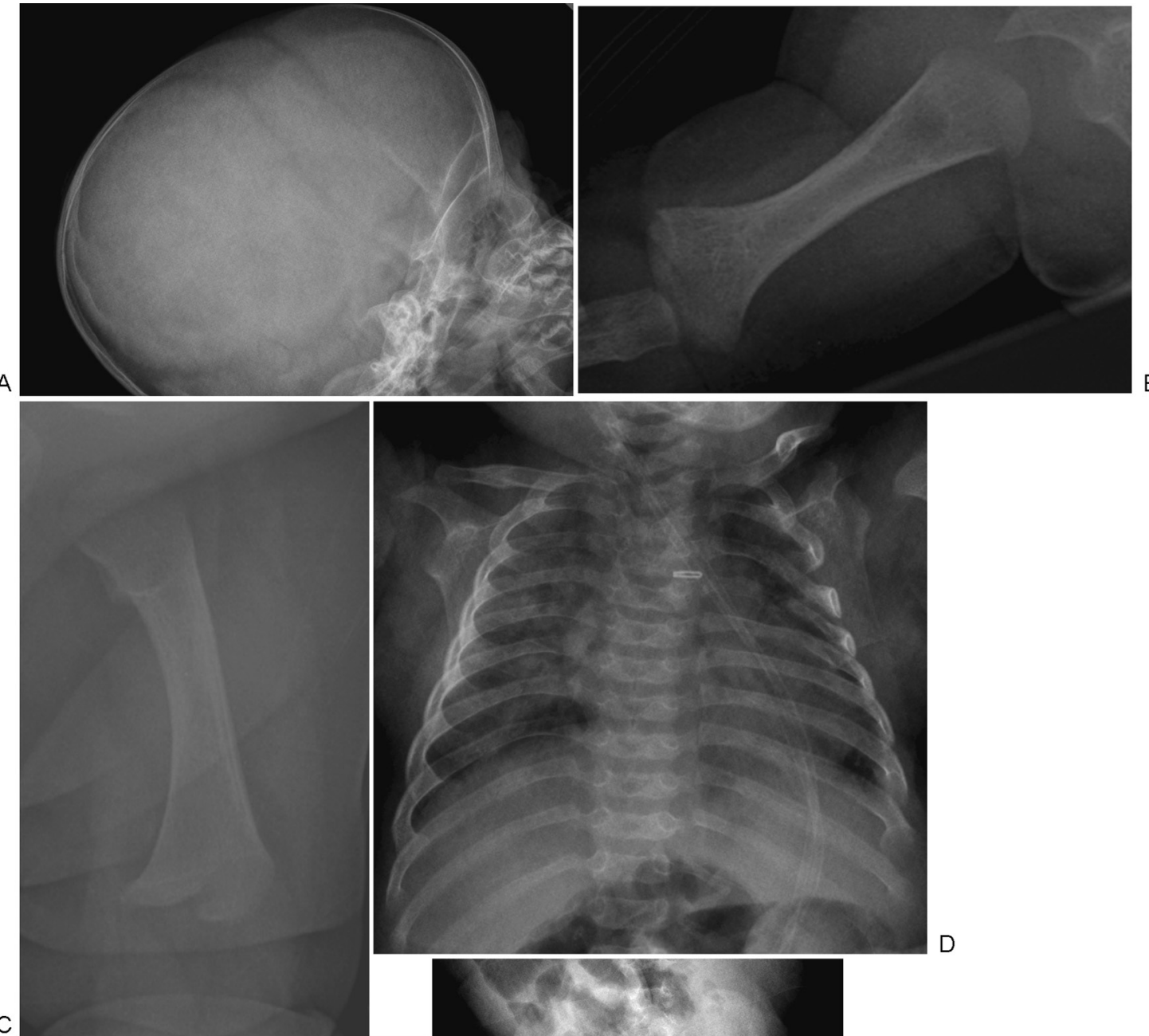

$\mathrm{C}$

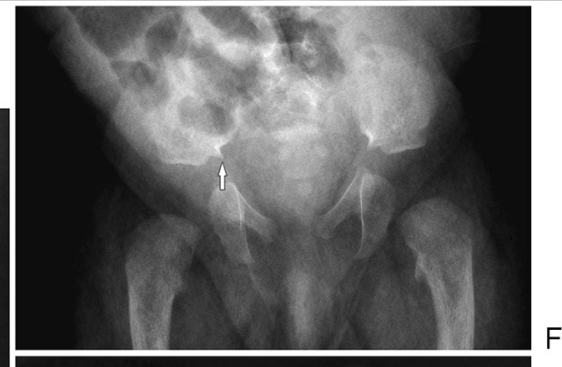

D

E

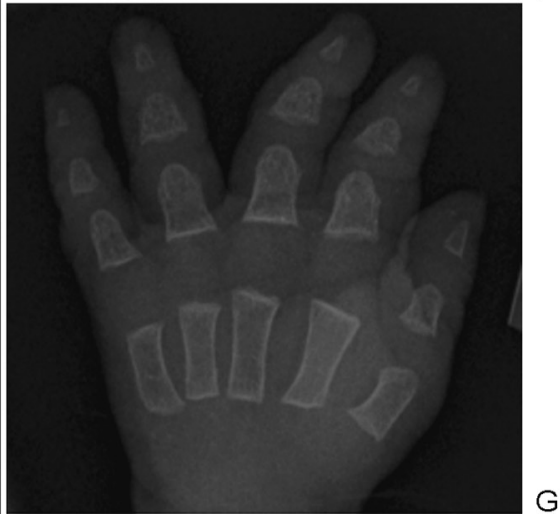

Fig. 1 Case 1: Skeletal survey. (A) Lateral skull radiograph shows frontal bossing, protuberance of the forehead which accentuates the craniofacial ratio. (B) Right humerus with rhizomelic (proximal limb) shortening and mild metaphyseal flaring, instead of smooth tapering of the diaphysis to the metaphysis. (C) Left femur with rhizomelic (proximal limb) shortening and metaphyseal flaring, more exaggerated widening of the metaphysis compared with the diaphysis, instead of smooth tapering in a normal long bone. (D) Chest frontal image shows small thorax with short, broadshaped ribs with some anterior flaring. (E) Lateral view of the spine demonstrates "bullet-shaped" vertebral bodies. Note that they appear wider than tall and some have anterior spurring. (F) Pelvis shows broad, squared ilia, narrow sacrosciatic notches, and a spur in the medial aspect of the flat acetabular roofs (arrow). (G) Trident hand with all phalanges short and broad. There is also widening of spacing between the middle and ring fingers. 
decreasing interpedicular distance particularly inferiorly in the lumbar spine, thoracic kyphosis with bullet-shaped vertebral bodies, trident hand with short and broad phalanges, and flat acetabular roofs with narrow sacrosciatic notches; these features were consistent with a diagnosis of achondroplasia.

Computed tomography (CT) was requested to rule out compression at the craniocervical junction and demonstrated craniocervical junction stenosis impinging on the brainstem and upper cervical cord (-Fig. 2). The craniofacial features of achondroplasia were also present including frontal bossing and diminutive midface. The presumptive radiological diagnosis of achondroplasia was confirmed by genetic DNA testing with a mutation in FGFR3 identified (c.1138 > Ap. Gly380Arg). The craniocervical junction stenosis was treated by craniovertebral decompression at 2 years of age.

Upon retrospective evaluation of the NICU imaging, subtle pelvic changes including narrowing of the sacrosciatic notches with medial spurs and flat acetabular roofs could be appreciated at 3 and 5 months of life (-Fig. 3). Narrowing of the interpedicular distances in the caudal portions of the lumbar spine was obscured by overlying bowel gas.

Case 2: This infant was born prematurely at 25 weeks' gestation due to maternal premature rupture of membranes. The infant was transferred to our NICU at 1 day of age and subsequently had a protracted course of bronchopulmonary dysplasia necessitating a tracheostomy. No clinical or radiological concerns for skeletal dysplasia were appreciated during the first few weeks of life. At 3 months of age, diaphyseal periostitis was noted in the humeri, thought to be most likely due to metabolic bone disease of prematurity but the possibility of a skeletal dysplasia was considered. The infant's flat nasal bridge with upturned nose, slight retrognathia, small hands, and short broad fingers were noted on evaluation by a geneticist, although the long bones were thought to appear proportional when visualized on abdominal radiographs. A

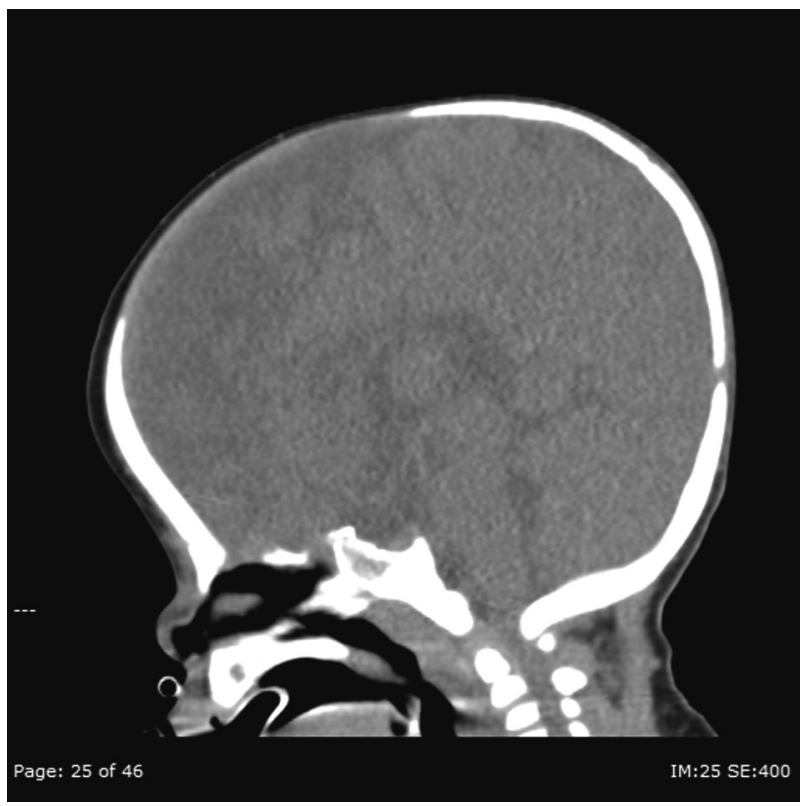

Fig. 2 Case 1: Computed tomography. Frontal bossing and severe narrowing of the foramen magnum with stenosis of the craniocervical junction.

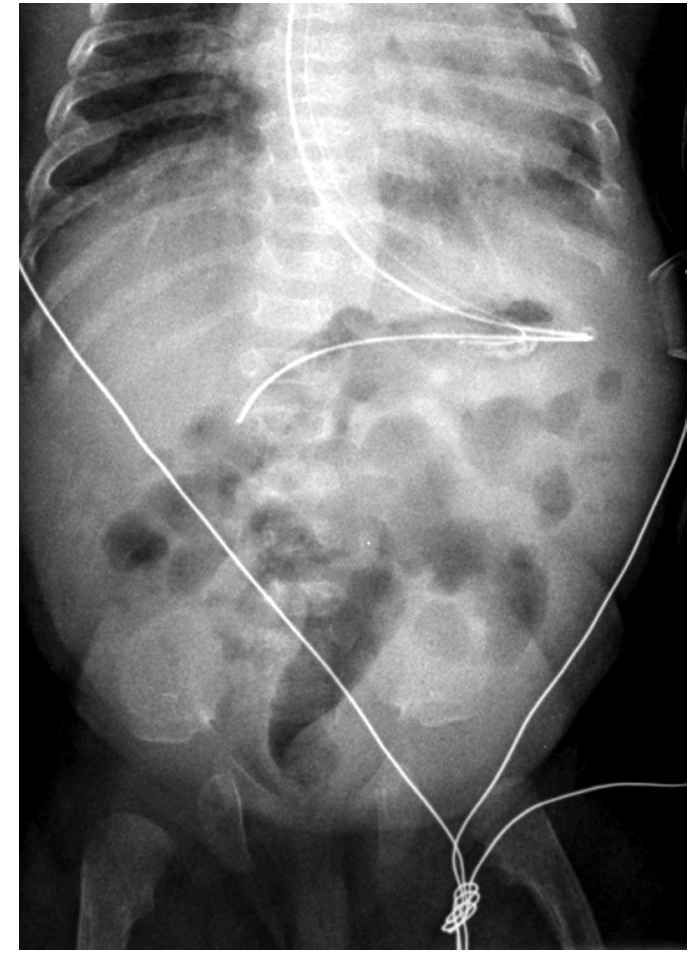

Fig. 3 Case 1: Portable abdominal radiograph in neonatal intensive care unit. Narrow sacrosciatic notch, flattened iliac wings, and medial acetabular roof spur with flat acetabulae can be retrospectively identified. Spine changes are not visible.

skeletal survey was suggested but deferred due to the patient's multiple medical problems.

Screening cranial ultrasound performed for prematurity demonstrated a midline cystic structure in the region of the velum interpositum. MRI at 5 months of age (term equivalent) to further characterize the cyst confirmed an arachnoid cyst in the velum interpositum, as well as fusion of the posterior intraoccipital synchondroses, severe stenosis of the foramen magnum, mild frontal bossing, ventriculomegaly, and hypoplasia of the mandible (-Fig. 4). The question of achondroplasia was raised and a skeletal survey was performed. The skeletal survey confirmed findings of achondroplasia. The thoracic cage was noted to be small. Bilateral coronal synostosis was also present. Genetic confirmation of the diagnosis was obtained with an FGFR3 molecular alteration.

In total, a 10 month NICU stay was required and the infant remained ventilator dependent with multiple medical problems.

Retrospective evaluation of NICU imaging confirmed the subtle pelvic changes of narrowing of the sacrosciatic notches with medial spurs and flat acetabular roofs could be appreciated as early as days 1 and 2 of life (-Fig. 5). Similar to case 1 , narrowing of the lumbar spine interpedicular distances caudally was difficult to visualize secondary to overlying bowel gas.

\section{Discussion}

Our two cases highlight the difficulty in the radiographic and clinical recognition of achondroplasia in the preterm infant. 


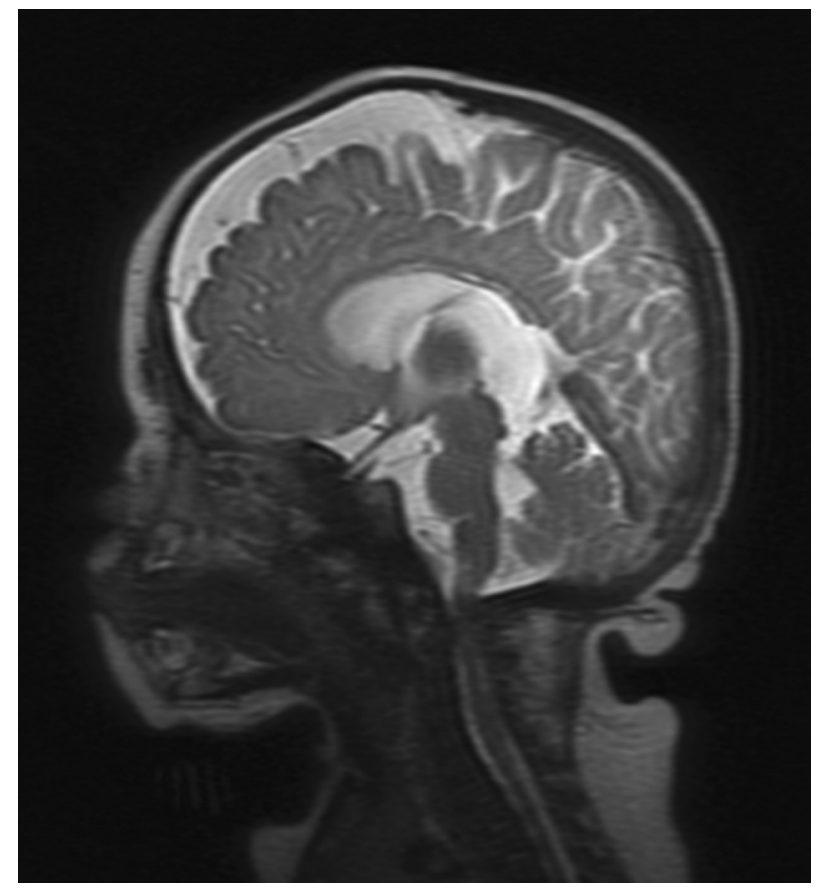

Fig. 4 Case 2: Magnetic resonance imaging. Sagittal image confirmed arachnoid cyst in the region of the velum interpositum and also shows mild frontal bossing, mandibular hypoplasia, and severe stenosis of the craniocervical junction.

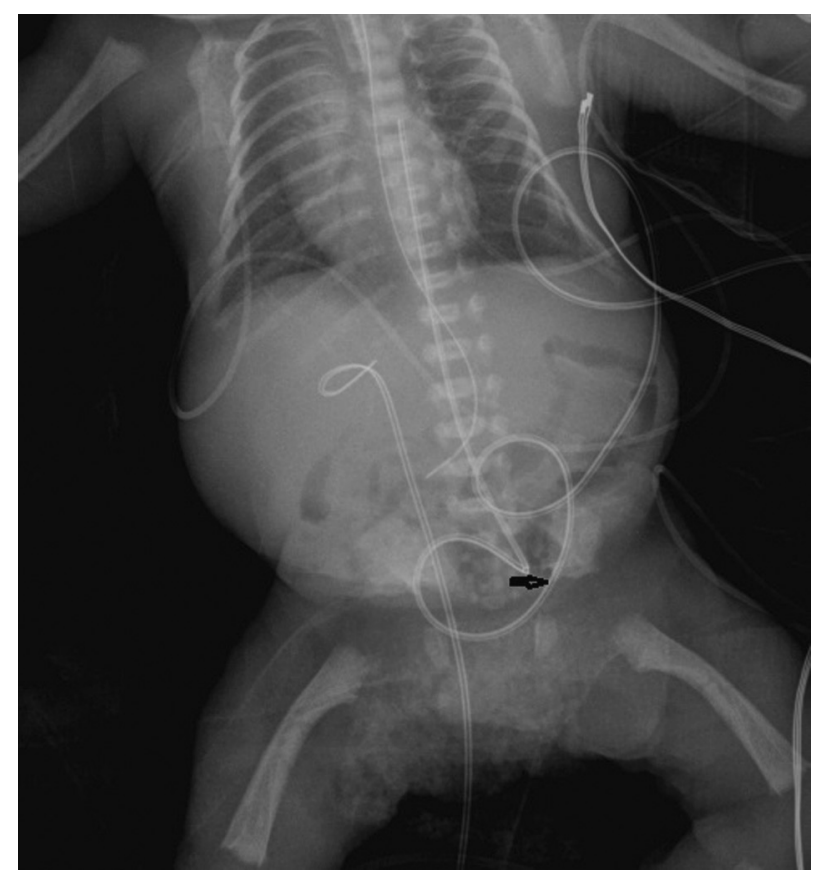

Fig. 5 Case 2: Portable abdominal radiograph in neonatal intensive care unit. Narrow sacrosciatic notches, medial acetabular roof spurs with flat acetabulae, flat square iliac wings, and mildly bowed proximal femora can be retrospectively identified. Note arrow on sacrosciatic notch which is difficult to see with overlying lines. Spine changes are not visible.
This difficulty mirrors the challenges of prenatal US and MRI diagnosis, with only subtle features present until the third trimester. Achondroplasia is typically not recognized on the second-trimester routine screening US. Therefore, this diagnosis may go unnoticed for many weeks or months in the premature infant. ${ }^{4,5}$

Subtle early US features include relative macrocephaly and larger abdominal circumferences with abnormal head circumference/femur length (HC/FL) and femur length/ abdominal circumference (FL/AC) ratios. ${ }^{1}$ In the early third trimester, limb shortening becomes more obvious when the femora and humeri begin to measure below the third percentile. $^{2}$

Frontal bossing, short and broad fingers, trident hand, and bowing of the femora (39\%) can also be recognized prenatally by US. ${ }^{1} \mathrm{~A}$ small chest and polyhydramnios may be seen in some fetuses. ${ }^{1}$ Prenatal three-dimensional CT has been used in the third trimester in some countries to confirm the diagnosis., ${ }^{2,3}$

Although DNA analysis is the gold standard for diagnosis, achondroplasia has classic postnatal radiographic findings that can be diagnostic. ${ }^{2-5}$ Skeletal survey long bone findings include rhizomelia, metaphyseal flaring, and mild bowing. Additional findings include frontal bossing of the calvarium, short fingers with widened space between the middle and ring fingers ("trident" hand), prominent medial sacrosciatic notches with spurs in the pelvis, "tombstone" square iliac bones, and "bullet-shaped" vertebral bodies with decreasing interpedicular distance of the caudal spine. Craniocervical stenosis may cause compression and kinking of the medullary cervical spine.

Thanatophoric dysplasia is a lethal dysplasia involving the FDFGR-3 with some overlap with achondroplasia in radiological features. Typically these cases present with severe micromelia in the early second trimester along with a small thorax, platyspondyly and markedly abnormal FL/AC. ${ }^{1,6}$ The early presentation makes the diagnosis easy to distinguish from nonlethal achondroplasia which presents with rhizomelic shortening in the later second trimester.

Our case analyses demonstrate that achondroplasia can be a difficult diagnosis to make before the third trimester both prenatally as well as postnatally, when these infants are born prematurely. In the setting of multiple pressing medical issues in early life, including bronchopulmonary dysplasia, $\mathrm{NEC}$, and poor feeding, the diagnosis may be easily overlooked in the preterm infant in the NICU. We have demonstrated the importance of careful radiographic osseous evaluation of premature infants for subtle skeletal signs of this elusive prenatal diagnosis. Recognition of pelvic changes may be most helpful in suggesting the diagnosis of achondroplasia in the preterm infant.

\section{References}

1 Chitty LS, Griffin DR, Meaney C, et al. New aids for the non-invasive prenatal diagnosis of achondroplasia: dysmorphic features, charts of fetal size and molecular confirmation using cell-free fetal DNA in maternal plasma. Ultrasound Obstet Gynecol 2011;37(3):283-289 
2 Boulet S, Althuser M, Nugues F, et al. Prenatal diagnosis of achondroplasia: new specific signs. Prenat Diagn 2009;29(7): 697-702

3 Yang P, Liao H, Yeh G, et al. Prenatal Diagnosis of achondroplasia with ultrasound, three-dimensional computed tomography and molecular methods. J Med Ultrasound 2012;20: 176-179
4 Unger S, Superti-Furga A, Rimoin D. A diagnostic approach to skeletal dysplasias. In: Glorieux F, Pettifor JM, Juppner H, eds. Pediatric Bone. 1st ed. Elsevier Science; 2003:375-400

5 Harris R, Patton JT. Achondroplasia and thanatophoric dwarfism in the newborn. Clin Genet 1971;2(2):61-72

6 Shah K, Astley R, Cameron AH. Thanatophoric dwarfism. J Med Genet 1973;10(3):243-252 